\title{
Combinatorial fluorescence energy transfer molecular beacons for probing nucleic acid sequences $\uparrow$
}

\author{
Xiaoxu Li, ${ }^{a}$ Zengmin Li, ${ }^{a, b}$ Angel A. Martí, ${ }^{c}$ Steffen Jockusch, ${ }^{c}$ Nathan Stevens, ${ }^{d}$ Daniel L. Akins, ${ }^{d}$ \\ Nicholas J. Turro ${ }^{* b, c}$ and Jingyue $\mathrm{Ju}^{* a, b}$
}

Received 26th April 2006, Accepted 28th June 2006

First published as an Advance Article on the web 12th July 2006

DOI: $10.1039 / \mathrm{b605936h}$

We report the design, synthesis, and characterization of molecular beacons (MB) consisting of three distinct fluorophores, 6-carboxyfluorescein (Fam), $N, N, N^{\prime}, N^{\prime}$-tetramethyl-6-carboxyrhodamine (Tam), and Cyanine-5 (Cy5). The primary light absorber/energy donor (Fam) is located on one terminus of the MB, whereas the primary energy acceptor/secondary donor (Tam) and secondary acceptor (Cy5) are located at the other terminus of the MB. In the absence of target DNA or RNA, the MB exists in the stem-closed form. Excitation of Fam initiates an energy transfer cascade from Fam to Tam and further to $\mathrm{Cy} 5$ generating unique fluorescence signatures defined as the ratio of the emission from each of the three fluorophores. This energy transfer cascade was investigated in detail by steady-state and time-resolved fluorescence spectroscopy, as well as fluorescence depolarization studies. In the presence of the complementary target DNA, the MB opened efficiently and hybridized with the target separating Fam and Tam by a large distance, so that energy transfer from Fam to Tam was blocked in the stem-open form. This opening of the MB generates a "bar code" fluorescence signature, which is different from the signature of the stem-closed MB. The fluorescence signature of this combinatorial fluorescence energy transfer MB can be tuned by variation of the spacer length between the individual fluorophores.

\section{Introduction}

Molecular beacons (MB) are single-stranded oligonucleotide probes which have shown high sensitivity and specificity in nucleic acid detection. ${ }^{1}$ In general, a $\mathrm{MB}$ is a single stranded oligonucleotide that forms a stem-loop structure, where the loop is complementary to the target sequence while the stem is labeled with a fluorescence sensor. ${ }^{2,3}$ "Classical" MBs possess a structure consisting of a fluorophore and a non-fluorescent quencher attached to opposite ends of a stem. In the stem-closed form of the MB the fluorescence of the sensor is quenched due to the close proximity of the quencher. In the presence of the target sequence of RNA or DNA the MB opens to form a double stranded structure. In this stem-open form, the fluorescent sensor and the quencher are separated, and strong fluorescence of the sensor is observed. ${ }^{4,5}$ MBs have found a wide range of applications such as genetic mutation detection, ${ }^{6}$ DNA/RNA target based pathogenic detection assays, ${ }^{7-12}$ DNA/RNA biosensors,${ }^{13}$ cellular imaging, ${ }^{14,15}$ DNA-protein interactions, ${ }^{1,16}$ and DNA cleavage assays. ${ }^{17}$

A variation to the "classical" $\mathrm{MB}$ is a $\mathrm{MB}$ where the quencher itself is fluorescent. ${ }^{18-20}$ This $\mathrm{MB}$ contains two fluorophores, a

${ }^{a}$ Columbia Genome Center, Columbia University College of Physicians and Surgeons, New York, NY, 10032

${ }^{b}$ Departments of Chemical Engineering, Columbia University, New York, NY, 10027

'Departments of Chemistry, Columbia University, New York, NY, 10027

${ }^{d}$ Department of Chemistry, City College, City University of New York, NY, 10031

$\dagger$ This paper was published as part of the special issue in honour of the late Professor George S. Hammond. fluorescent donor and a fluorescent acceptor, which are covalently attached at opposite termini of the stem. In the stem-closed form the donor fluorescence is quenched by the acceptor through fluorescence resonance energy transfer (FRET), generating predominantly fluorescence of the acceptor, which is at a different wavelength from the fluorescence of the donor. In the stem-open form, FRET is negligible and mainly the fluorescence of the selectively excited donor is observed. For practical applications a large spectral separation between the donor and acceptor fluorescence is desired. The large spectral separation minimizes the spectral overlap of donor and acceptor absorption, reducing competitive absorption of the light and reduces interference between the donor and acceptor fluorescence. In microscopy, this allows for the use of bandpass filters with a wider spectral bandpass for the two detection channels, which enhances the signal intensity significantly. However, a large spectral separation of the donor and acceptor dyes can reduce the FRET efficiency. In practice, it is difficult to find a pair of dyes that fulfill the condition of low direct excitation of the acceptor dye and high FRET efficiency. However, one of our previous studies illustrated that a covalent assembly of a triple fluorophore FRET system dramatically enhances acceptor emission with the advantage of a large "Stoke's shift". ${ }^{21}$ In addition, adjustment of the distances between a small number of fluorophores produces a large number of unique combinatorial fluorescence energy transfer tags with distinguishable fluorescence energy transfer "bar code" signatures, which have been successfully used in multiplex detection of singlenucleotide polymorphisms (SNPs). ${ }^{22,23}$

Here we report the design, synthesis, and characterization of a combinatorial fluorescence energy transfer molecular beacon 


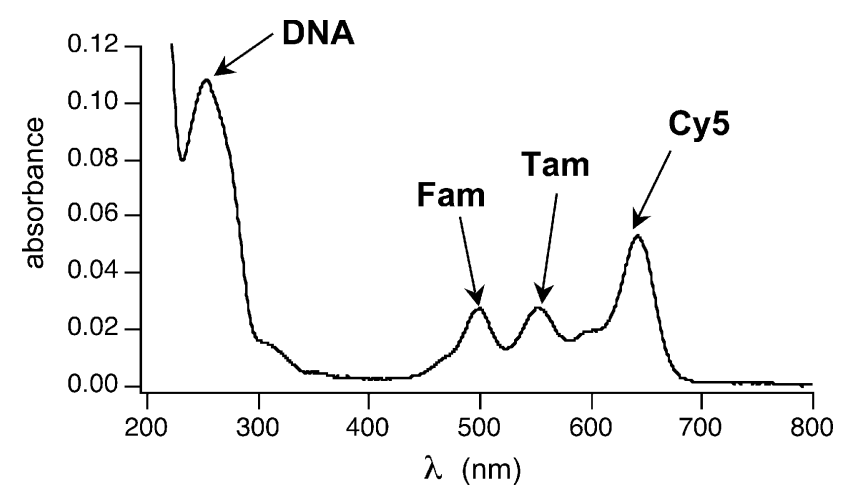

Fig. 1 Absorbance spectrum of $\mathrm{MB}(0,6)$ in a solution of $50 \%$ acetonitrile in water.

(CFET-MB) system (Scheme 1) in which a donor fluorophore 6carboxyfluorescein (Fam) is linked at the $3^{\prime}$ end, while two more acceptor fluorophores $N, N, N^{\prime}, N^{\prime}$-tetramethyl-6-carboxyrhodamine (Tam) and Cyanine-5 (Cy5) are linked to the other end of the $\mathrm{MB}$. When the MB is in the stem-closed configuration, excitation of Fam (488 nm) promotes an energy transfer cascade that goes from Fam to Tam to Cy5 where the energy is released as fluorescence emission of Cy5 (670 nm) (Scheme 1, left). Upon hybridization of the CFET-MB with the target DNA, Fam and the Tam-Cy 5 pair are separated, and the fluorescence occurs preferentially from the selectively excited Fam donor (Scheme 1, right). The distance $\left(S_{\mathrm{n}}\right)$ between the fluorophores was systematically varied using 1,2-dideoxyribose phosphate spacers. ${ }^{22,23}$ The synthesized MBs with different spacers are labeled $\operatorname{MB}(m, n)$, where $m$ and $n$ are the number of spacers and are defined in Fig. 2 a.

In order to test the concept of CFET-MBs we selected the loop and stem sequence, which was successfully used previously for a FRET MB containing two fluorophores for nucleic acid target detection. ${ }^{18}$ The loop sequence was selected based on our interest to study sensorin mRNA in neurons of the mollusk Aplysia Californica. ${ }^{24}$

\section{Experimental}

\section{Synthesis of the MBs}

The MBs (Scheme 1) were prepared by solid-phase phosphoramidite chemistry on a DNA/RNA synthesizer (Expedite 8909, Applied Biosystems, CA, USA). A set of iBu$\mathrm{dA}$, iPr-Pac-dG, Ac-dC and dT-CE phosphoramidites along with dSpacer (5-O-dimethoxytrityl-1-2-dideoxyribose-CE phosphoramidite), Fam-dT (6-carboxyfluorescein derivative) phosphoramidite, Tam-dT-CE phosphoramidite $\left(N, N, N^{\prime}, N^{\prime}\right.$-tetramethyl6-carboxyrhodamine derivative), and Cy5-CE phosphoramidite (Cyanine-5 derivative) from Glen Research (Sterling, VA, USA) were used. Because TAMRA is sensitive to ammonium hydroxide treatment (the most commonly used method for cleavage of the oligonucleotide from the CPG support and deprotection) a milder condition was used. As recommended by Glen Research (Sterling, VA, USA), the cleavage of the fluorescent oligonucleotides from the support and deprotection were performed with $1 \mathrm{ml} 0.05 \mathrm{M}$ $\mathrm{K}_{2} \mathrm{CO}_{3}$ in methanol for $6 \mathrm{~h}$ at room temperature. The crude products were desalted by OPC (Applied Biosystems, CA, USA). The dried crude samples were then purified by reverse-phase HPLC (Waters system containing a Waters Delta 600 controller, Rheodyne 7725i injector, and 2996 photodiode array detector, Milford, MA, USA) using C-18 reverse column (Xterra MS $\mathrm{C} 18 ; 4.6 \mathrm{~mm} \times 50 \mathrm{~mm} ; 2.5 \mu \mathrm{m}, 300 \AA$ ) at a flow rate of $0.5 \mathrm{ml} \mathrm{min} \mathrm{m}^{-1}$, with detection at 260,550 and $650 \mathrm{~nm}$, and elution with a linear gradient of $12-34.5 \%$ of $\mathrm{B}$ in A over 40 min (A: $8.6 \mathrm{mM}$ triethylammonium and $100 \mathrm{mM}$ hexafluoroisopropyl
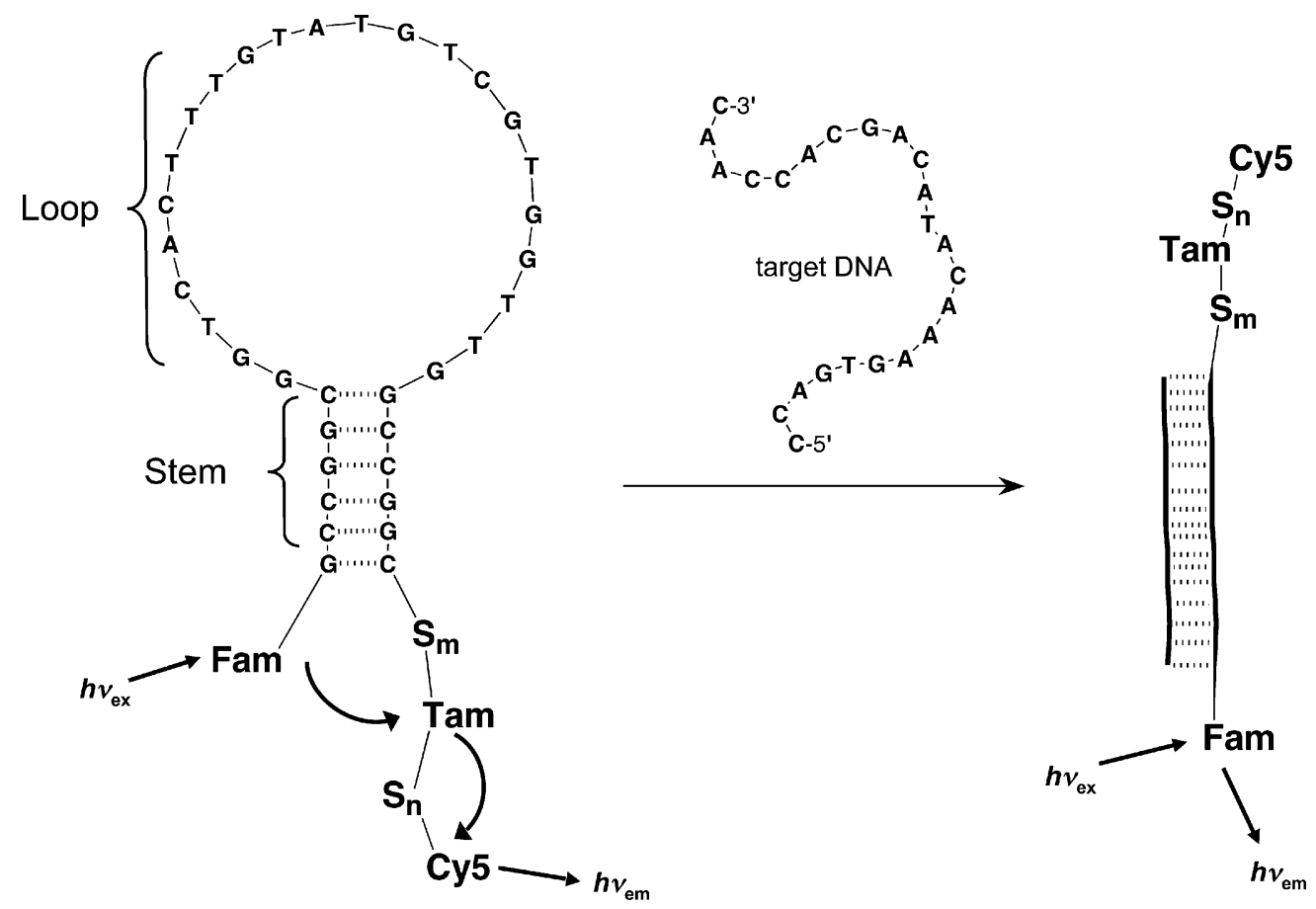

Scheme 1 Schematic structure of the CEFT-MB in its stem-closed (left) and stem-open form (right). 

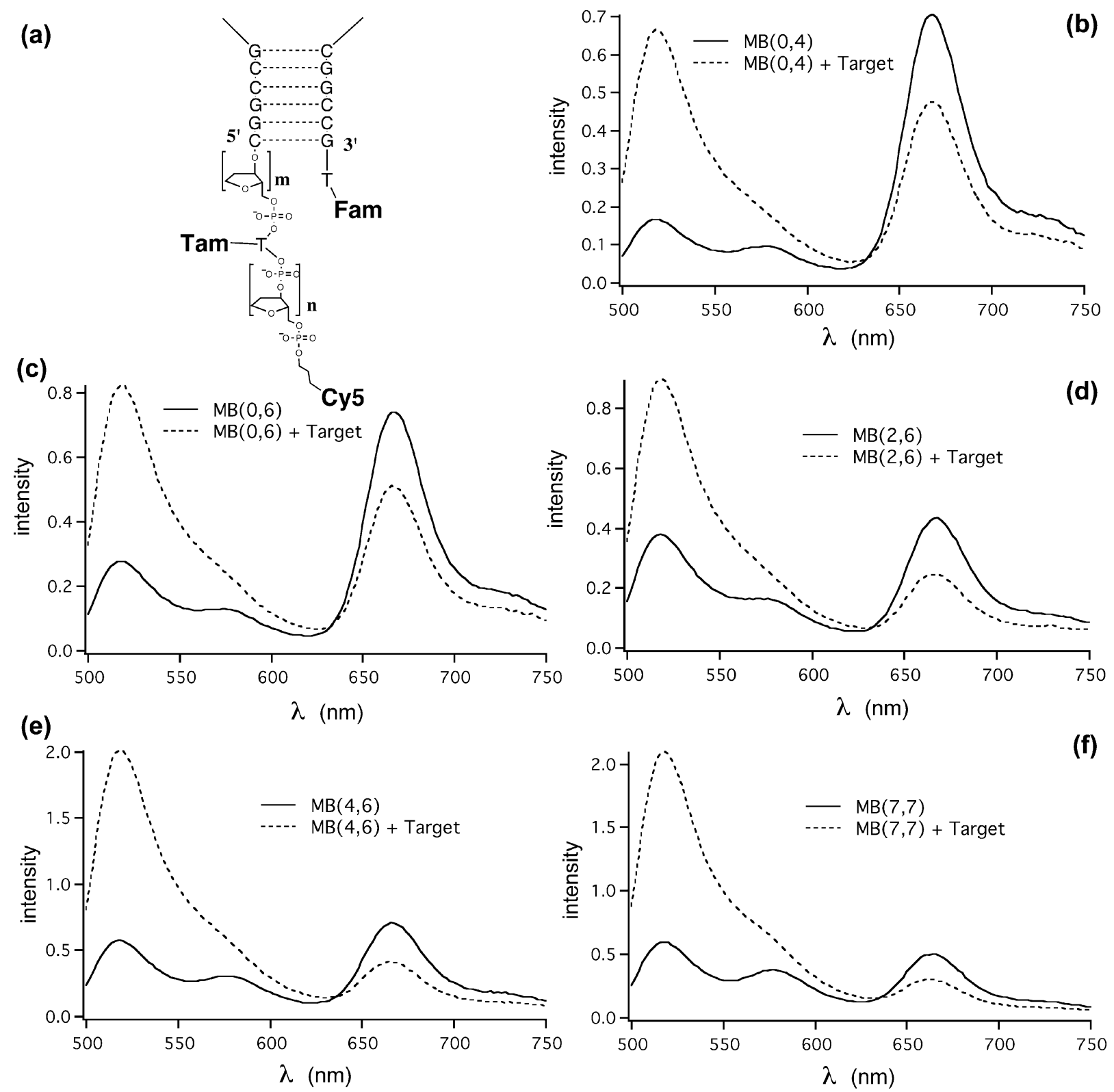

Fig. 2 Structure of CFET-MB $(m, n)$, where $m$ and $n$ denote the number of spacers between the fluorophores (a). Fluorescence spectra (b-f) after excitation $(488 \mathrm{~nm})$ of $\mathrm{MB}(m, n)(0.3 \mu \mathrm{M})$ in aqueous buffer solution $(20 \mathrm{mM}$ Tris, $5 \mathrm{mM} \mathrm{MgCl} 250 \mathrm{mM} \mathrm{NaCl}$; $\mathrm{pH}=7.6)$ at $488 \mathrm{~nm}$ in the absence $(\longrightarrow)$ and presence (---) of target $(3 \mu \mathrm{M})$.

alcohol aqueous solution, $\mathrm{pH}$ 8.1; B: methanol). The purified MBs were identified by MALDI-TOF mass spectrometry (MS) using a Voyager DE mass spectrometer (Applied Biosystems): $\mathrm{MB}(0,4)$ 13770 (found), 13717 (calculated); $\mathrm{MB}(0,6) 14129$ (found), 14076 (calculated); $\operatorname{MB}(2,6) 14488$ (found), 14434 (calculated); $M B(4,6)$ 14842 (found), 14792 (calculated); $\operatorname{MB}(7,7) 15561$ (found) 15508 (calculated).

\section{Synthesis of the target DNA}

The target DNA (Scheme 1) was synthesized by standard solidphase phosphoramidite chemistry on a DNA synthesizer (Expetide 8909; Applied Biosystems). The obtained crude product was deprotected by concentrated $\mathrm{NH}_{4} \mathrm{OH}$, purified by OPC (Oligonu- cleotide Purification Cartridge, Applied Biosystems, Foster, CA, USA) and RP-HPLC, and was characterized by MALDI-TOF MS: 6975 (calculated), 6978 (found).

\section{UV-Vis absorption spectroscopy}

Absorbance spectra were recorded on a Lambda 40 spectrophotometer (Perkin Elmer, Norwalk, CT, USA) at room temperature using quartz cuvettes with a path length of $1 \mathrm{~cm}$.

\section{Steady-state fluorescence and polarization measurement}

Fluorescence emission spectra and polarization values were recorded at room temperature on a SPEX Fluorolog-3 spectrometer FL3-22 (J. Y. Horiba, Edison, NJ, USA) using quartz cells with 
path length of $4 \mathrm{~mm}$ and Glan Thompson polarizers, respectively. The spectra were corrected for detector response and grating efficiency according to the correction factor file pertaining to the instrument. The polarization values $(P)$ for the individual dye components and the energy transfer components were determined by exciting one dye with linear polarized light and analyzing the depolarization of the fluorescence at different wavelengths for the two fluorophores.

\section{Time-resolved fluorescence measurement}

Time-dependent emission measurements utilized a Hamamatsu streak camera, Model C4334, optically coupled to a chargecoupled-device (CCD) array detector. This system allowed the measurements of both the emission decay and the time-resolved emission spectrum. For this latter study a Chromex 205i imaging spectrometer was used. The excitation source for these studies was an all-solid-state laser system from Spectra Physics, which incorporates the following components: a diode-pumped Millennia V-P laser for exciting mode-locked lasing from a Tsunami (Model 3941M1S) Ti-sapphire laser, which in turn was amplified by a Spitfire regenerative amplifier with a Merlin regenerative pump source. Tunable, femtosecond radiation was acquired through the use of an optical parametric amplifier (OPA-800P) in combination with harmonic generation and sum-difference packages. The tunable laser pulses were of $c a .150 \mathrm{fs}$ in duration at a repetition rate of 1 $\mathrm{kHz}$. For the present study, 488 and $550 \mathrm{~nm}$ pulses were used to excite the CFET-MB system. The ultimate time resolution that we have been able to attain with this system, using Hamamatsu U4290 fluorescence analysis software, was estimated to be $\sim 10 \mathrm{ps}$.

\section{Results and discussion}

Fig. 1 shows a typical absorbance spectrum of the threefluorophore CFET-MB, where absorption peaks at 260, 496, 555, $643 \mathrm{~nm}$ are observed corresponding to the absorption of the nucleotides $\left(\varepsilon=7050-15200 \mathrm{M}^{-1} \mathrm{~cm}^{-1}\right), \operatorname{Fam}\left(\varepsilon=75000 \mathrm{M}^{-1} \mathrm{~cm}^{-1}\right)$, Tam $\left(\varepsilon=89000 \mathrm{M}^{-1} \mathrm{~cm}^{-1}\right)$, and Cy5 $\left(\varepsilon=250000 \mathrm{M}^{-1} \mathrm{~cm}^{-1}\right)$, respectively. Steady-state fluorescence analysis of the MBs was performed at an excitation wavelength of $488 \mathrm{~nm}$, where Fam predominantly absorbs. Fig. 2b-f show the fluorescence spectra (with maxima at $518 \mathrm{~nm}, 576 \mathrm{~nm}$, and $666 \mathrm{~nm}$ ) of the CFETMBs. Excitation of the stem-closed $\mathrm{MB}(0,4)$ at $488 \mathrm{~nm}$ excites Fam preferentially; however the fluorescence spectrum is dominated by a strong signal at $666 \mathrm{~nm}$ corresponding to Cy5, suggesting an energy hopping mechanism in which Fam acts as a primary donor, Tam as a primary energy acceptor-secondary donor and Cy5 as the final energy acceptor releasing the energy as fluorescence with a peak at $666 \mathrm{~nm}$ (Fig. 2b). An increase in the distance between Tam and Cy5 $[\mathrm{MB}(0,6)]$ causes a decrease in energy transfer efficiency, and the ratio between the fluorescence intensity of Tam to Cy5 increased (Fig. 2c) compared to $\mathrm{MB}(0,4)$ (Fig. 2b). In addition, increasing the distance between Fam and $\operatorname{Tam}[\mathrm{MB}(0,6)$ to $\mathrm{MB}(2,6)$ to $\mathrm{MB}(4,6)$ to $\mathrm{MB}(7,7)$ ] causes a further increase in the ratio between the fluorescence intensity of Tam relative to $\mathrm{Cy} 5$ indicating a decrease in the FRET efficiency. Table 1 summarizes the fluorescence ratios of the dyes for the MBs with different spacer lengths. Both fluorescence intensity ratios, $I_{\mathrm{Cy} 5} / I_{\mathrm{Fam}}$ and $I_{\mathrm{Cy} 5} / I_{\mathrm{Tam}}$, correlate well with the spacer lengths for all the investigated MBs.
Table 1 Fluorescence signature of stem-closed CFET-MBs with different spacer lengths between the fluorophores $(1 \mu \mathrm{M} \mathrm{MB}$ in buffer solution, $\lambda_{\mathrm{ex}}=488 \mathrm{~nm}$ )

\begin{tabular}{lll}
\hline Molecular beacon & $I_{\mathrm{Cy} 5} / I_{\mathrm{Fam}}{ }^{a}$ & $I_{\mathrm{Cy} 5} / I_{\mathrm{Tam}}{ }^{b}$ \\
\hline $\operatorname{MB}(0,4)$ & 4.2 & 7.4 \\
$\operatorname{MB}(0,6)$ & 2.7 & 5.8 \\
$\operatorname{MB}(2,6)$ & 1.1 & 2.6 \\
$\operatorname{MB}(4,6)$ & 1.2 & 2.3 \\
$\operatorname{MB}(7,7)$ & 0.87 & 1.3
\end{tabular}

${ }^{a}$ Fluorescence intensity ratio between Cy5 and Fam $\left(\lambda_{\text {monitor }}=666 \mathrm{~nm}\right.$, $\lambda_{\text {monitor }}=518 \mathrm{~nm}$, respectively). ${ }^{b}$ Fluorescence intensity ratio between Cy 5 and $\operatorname{Tam}\left(\lambda_{\text {monitor }}=666 \mathrm{~nm}, \lambda_{\text {monitor }}=576 \mathrm{~nm}\right.$, respectively $)$.

The modulation of interchromophoric distances provides a tool to tune the FRET efficiency, providing a unique fluorescent signature for each different configuration.

Addition of the target oligonucleotide and hybridization with the MB increase the spatial separation of the fluorophores located at different ends of the MB strand, which precludes the energy transfer cascade. The net result is the recovery of Fam fluorescence at $518 \mathrm{~nm}$ and the blocking of the FRET emission at $666 \mathrm{~nm}$ as shown in Fig. 2 (dashed curves). However, some Cy5 emission is still observed on the spectra due to some residual absorption of Cy5 and Tam even at $488 \mathrm{~nm}$.

In order to investigate the energy transfer dynamics, fluorescence lifetime studies were performed using pulses from a Ti-sapphire laser system (488 nm, 150 fs pulse duration) in conjunction with a streak camera. Fig. 3 shows the fluorescence dynamics of $\mathrm{MB}(4,6)$ as an example. Excitation of Fam of the stem-closed $\mathrm{MB}(4,6)$ generated fluorescence of Fam, Tam, and Cy5 within a time less than 100 ps after the laser pulse (Fig. 3a). The temporal growths of Tam and $\mathrm{Cy} 5$ are slightly delayed relative to that of Fam, which is consistent with a delayed energy transfer from Fam to Tam followed by energy transfer to Cy5. This delayed energy transfer is consonant with prior energy transfer studies of these three fluorophores covalently linked to DNA, where the fluorophores were separated by a few bases. ${ }^{21}$ The decay dynamics are shown in Fig. 3b, which were fitted to biexponential decay functions. The lifetimes and the corresponding fluorescence intensity contributions are listed in Table 2. In case of stemopen $\operatorname{MB}(4,6)$, the growth dynamics of all three dye emissions are essentially identical (Fig. 3c), which indicates that the fluorophores are excited directly and not through energy transfer. The decay dynamics of the stem-open $\mathrm{MB}(4,6)$ are shown in Fig. $3 \mathrm{~d}$ and the fluorescence lifetimes are listed in Table 2. The slower fluorescence decay of Fam in the stem-open MB compared to the stemclosed form is consistent with the inhibited energy transfer in the stem-open form. The decay dynamics of the stem-open $\operatorname{MB}(4,6)$ monitored from 570-600 nm (max. fluorescence of Tam) and 650$690 \mathrm{~nm}$ (Cy5) are almost identical (Fig. 3d). This observation, coupled with the absence of a clearly discernible fluorescence contribution by Tam to the steady-state stem-open fluorescence spectrum (Fig. 2e), is consistent with the dynamics of the weak fluorescence monitored from $570-600 \mathrm{~nm}$ as being dominated by the fluorescence of Fam.

To further study the fluorescence dynamics of the dyes in $\mathrm{MB}(4,6)$, fluorescence experiments were performed by exciting Tam directly at $550 \mathrm{~nm}$. The temporal traces are shown in 
Table 2 Photophysical parameters of stem-closed (absence of target) and stem-open (presence of target; $10 \mu \mathrm{M})$ of $\mathrm{MB}(4,6)(1 \mu \mathrm{M})$

\begin{tabular}{|c|c|c|c|c|c|}
\hline Absence $(-) /$ presence of target & Excitation/emission $^{a}$ & 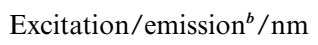 & $\tau_{1} /$ ns $[\text { contribution }(\%)]^{c}$ & $\tau_{2} / \mathrm{ns}[\text { contribution }(\%)]^{c}$ & $P^{d}$ \\
\hline- & $\mathrm{Fam} / \mathrm{Fam}$ & $488 / 515$ & $2.5[18]$ & $0.24[82]$ & 0.11 \\
\hline Target & $\mathrm{Fam} / \mathrm{Fam}$ & $488 / 515$ & $3.1[78]$ & $0.61[22]$ & 0.07 \\
\hline - & Fam/Tam & $488 / 559$ & $2.6[30]$ & $0.27[70]$ & 0.06 \\
\hline Target & Fam/Tam & $488 / 559$ & $2.9[77]$ & $0.53[23]$ & 0.08 \\
\hline- & Fam/Cy 5 & $488 / 650$ & $1.3[47]$ & $0.41[53]$ & 0.005 \\
\hline Target & Fam/Cy5 & $488 / 650$ & $1.7[40]$ & $0.51[60]$ & 0.02 \\
\hline - & Tam/Tam & $550 / 559$ & $2.2[22]$ & $0.25[78]$ & 0.21 \\
\hline Target & Tam/Tam & $550 / 559$ & $2.3[43]$ & $0.35[57]$ & 0.19 \\
\hline - & Tam/Cy5 & $550 / 660$ & $1.2[43]$ & $0.38[57]$ & 0.11 \\
\hline Target & Tam/Cy 5 & $550 / 660$ & $1.3[45]$ & $0.51[55]$ & 0.09 \\
\hline- & Сy $5 /$ Cy 5 & $559 / 670$ & $1.7[48]$ & $0.72[52]$ & 0.33 \\
\hline Target & Сy5/Cy5 & $559 / 670$ & $1.7[49]$ & $0.75[51]$ & 0.31 \\
\hline
\end{tabular}

${ }^{a}$ Excitation/emission of the individual dye ${ }^{b}$ Excitation wavelength/emission wavelength of the individual dye. ${ }^{c}$ Fluorescence lifetime component $(\tau)$ and corresponding fluorescence intensity contribution $(\%) .{ }^{d}$ Polarization value.
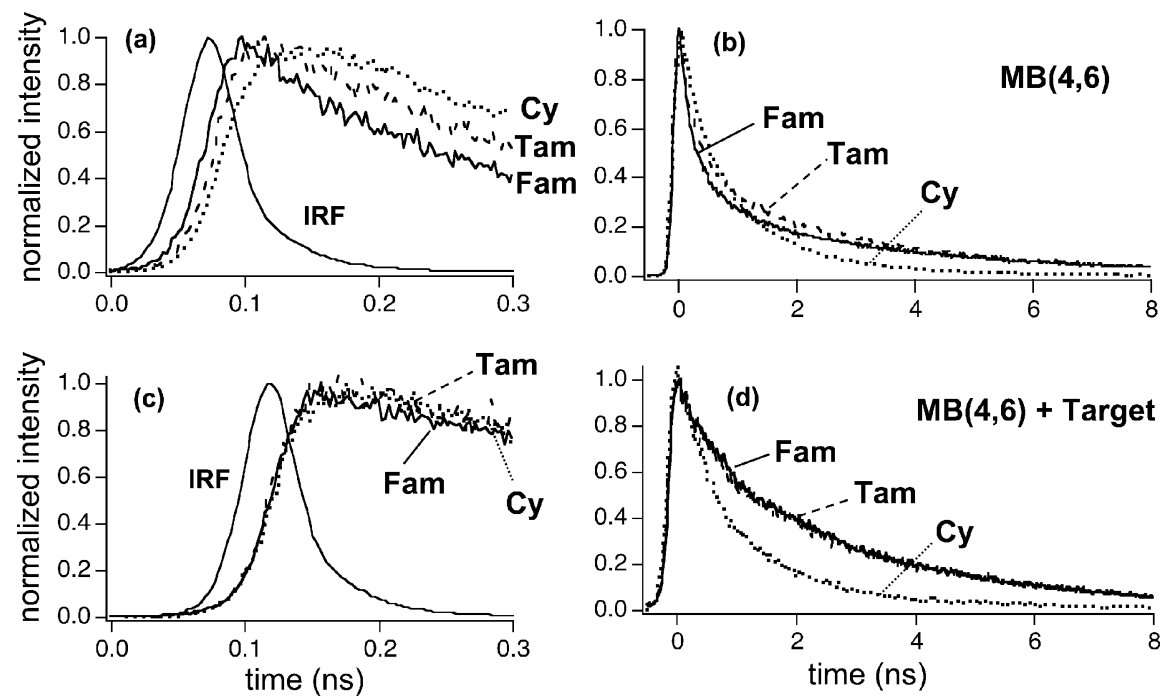

Fig. 3 Fluorescence decays after laser excitation ( $488 \mathrm{~nm}, 150 \mathrm{fs}$ pulses) of $\mathrm{MB}(4,6)(1 \mu \mathrm{M})$ in aqueous buffer solutions in the absence (a, b) and presence of the target (c, d) monitored from 510-530 nm (Fam), 570-600 nm (Tam), and 650-690 nm (Cy5). The temporal response function of the instrument, labeled here as IRF is shown in $(\mathrm{a}, \mathrm{c})$.

Fig. 4 and the lifetimes after biexponential decay fitting are listed in Table 2. As expected, no major difference in the dynamics was observed between the stem-closed and stem-open $\mathrm{MB}(4,6)$. The slightly delayed fluorescence rise of Cy5 compared to Tam indicates slow energy transfer from Tam to Cy5 (Fig. 4a,c). The slow energy transfer makes it possible to observe distinct fluorescence signatures, where the donor dyes still show some fluorescence. In control experiments, the fluorescence lifetime of the acceptor dye, Cy5, was determined by direct excitation of $\mathrm{Cy} 5$ at $659 \mathrm{~nm}$. As expected, almost no difference in the decay dynamics was observed between the stem-closed and stem-open $\operatorname{MB}(4,6)$ (Table 2).

A more thorough analysis of the fluorescence decay dynamics is very challenging to perform, because of the complexity of the involved quenching mechanisms. In addition to the above discussed FRET mechanisms, quenching by the nucleotides and competitive absorption of the excitation light by the acceptors contributed to the complex fluorescence dynamics.

Fluorescence depolarization studies provided further information for the photophysics of $\mathrm{MB}(4,6)$. Table 2 shows the polarization values $(P)$ for the individual fluorophores and energy transfer components. The $P$ values were determined by exciting one fluorophore with linear polarized light and analyzing the depolarization of the fluorescence at different wavelength corresponding to the individual fluorophores. A fluorophore can lose its polarization by rotation during its fluorescence lifetime..$^{25}$ Therefore, lower polarization values are observed for longer fluorescence lifetimes. Our observed polarization values are in good agreement with the observed fluorescence lifetimes. For example, in the stem-closed $\mathrm{MB}(4,6)$ a polarization value for Fam after excitation of Fam of $P_{\text {FamFam }}=0.11$ was observed, however, in the stem-open $\mathrm{MB}(4,6)$, where the fluorescence lifetime of Fam is longer, the polarization value is smaller $\left(P_{\text {FamFam }}=0.07\right)$ (Table 2$)$. The polarization is partly preserved during fluorescence energy transfer. For example, in the stem-closed $\mathrm{MB}(4,6)$ a polarization value for Tam after excitation of Fam and energy transfer to Tam of $P_{\text {FamTam }}=0.06$ was observed. In the second energy transfer step from Tam to Cy5, a small amount of polarization is preserved $\left(P_{\text {FamCy }}=0.005\right)$. The polarization value of Cy5 for direct excitation of $\mathrm{Cy} 5$ in the stem-closed $\mathrm{MB}(4,6)$ is significantly 

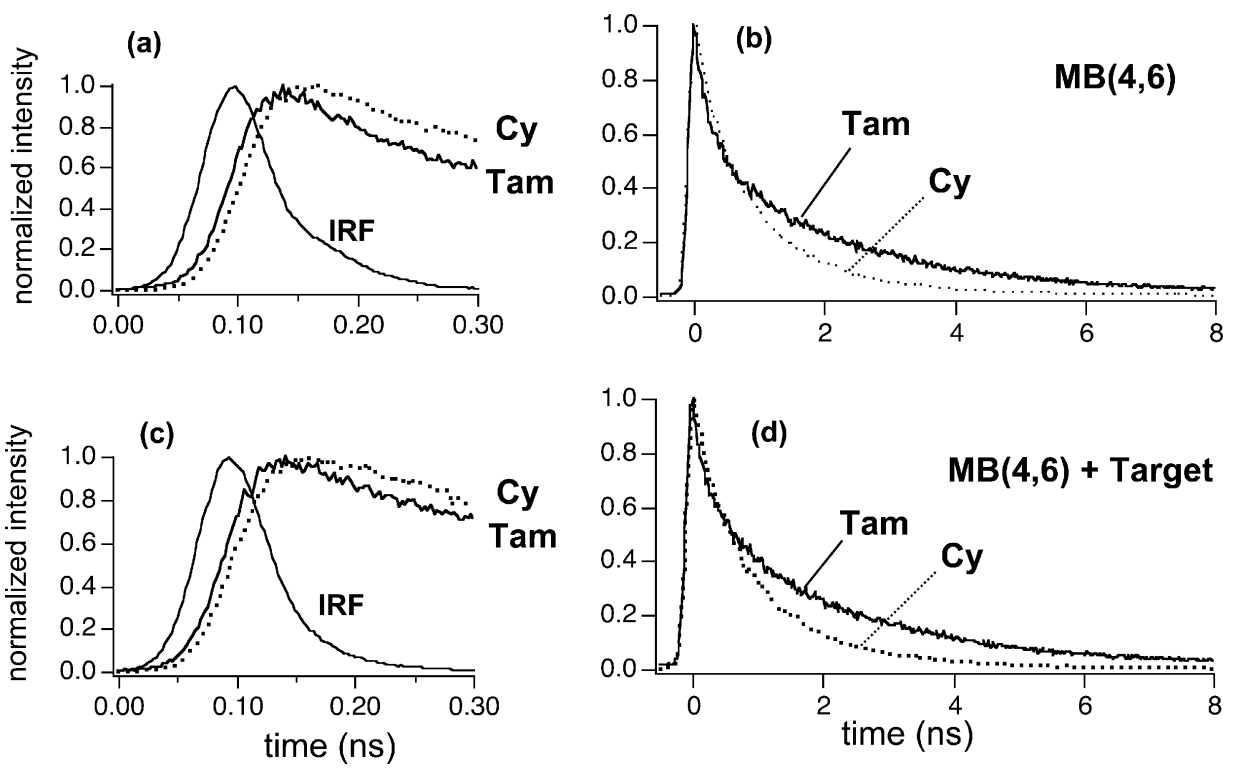

Fig. 4 Fluorescence decays after laser excitation ( $550 \mathrm{~nm}, 150 \mathrm{fs}$ pulses) of $\mathrm{MB}(4,6)(1 \mu \mathrm{M})$ in aqueous buffer solutions in the absence (a, b) and presence of the target (c, d) monitored from 570-600 nm (Tam), and 650-690 nm (Cy5). The temporal response function of the instrument, labeled here as IRF is shown in $(a, c)$.

larger $\left(P_{\mathrm{C} y 5 \mathrm{C} y 5}=0.33\right)$. If the fluorescence of $\mathrm{Cy} 5$ is generated by energy transfer from Tam, the polarization value is lower $\left(P_{\text {TamCy }}=\right.$ $0.11)$, and even lower after double energy transfer from Fam to Tam and further to Cy5 $\left(P_{\text {FamCy } 5}=0.005\right)$.

\section{Conclusions}

This study shows that CFET tags can be used as the fluorescent reporter in MBs. With a limited number of individual fluorescent molecules, a larger number of fluorescent labels can be produced, which provides a new "bar code" approach for multiple targets detection by using MB probes. Furthermore, a major advantage of a three-fluorophore CFET-MB compared to conventional twofluorophore energy transfer MBs is the large spectral separation between excitation wavelength and final fluorophore emission. This large "Stoke's shift" minimizes the spectral overlap of donor and acceptor dye absorption, reducing competitive absorption of the light, and reduces interference between the donor and acceptor fluorescence. For microscopy imaging, this large "Stoke's shift" allows for the use of bandpass filters with a wider spectral bandpass of the two detection channels, which will enhance the signal intensity significantly.

\section{Acknowledgements}

This work was supported by the Center of Excellence in Genomic Science Grant P50 HG002806 from the National Institutes of Health, NSF CHE-04-15516 and by NSF CHE-04-13574. DLA thanks the NSF and DoD-ARO for support of this work, in part, through the following awards: (1) the NSF-IGERT Program, under Grant No. DGE-9972892; (2) the NSF-MRSEC Program, under Grant No. DMR-0213574; and (3) DoD-ARO, under Cooperative Agreement DAAD19-01-1-0759.

\section{References}

1 W. Tan, X. Fang, J. Li and X. Liu, Molecular beacons: a novel DNA probe for nucleic acid and protein studies, Chem.-Eur. J., 2000, 6, 11071111.

2 G. Bonnet, S. Tyagi, A. Libchaber and F. R. Kramer, Thermodynamic basis of the enhanced specificity of structured DNA probes, Proc. Natl. Acad. Sci. U. S. A., 1999, 96, 6171-6176.

3 T. Antony and V. Subramaniam, Molecular Beacons: Nucleic Acid Hybridization and Emerging Applications, J. Biomol. Struct. Dyn., 2001, 19, 4997-5004.

4 S. Tyagi and F. R. Kramer, Molecular Beacons: Probes that Fluoresce upon Hybridization, Nat. Biotechnol., 1996, 14, 303-308.

5 S. Tyagi, D. P. Bratu and F. R. Kramer, Multicolor molecular beacons for allele discrimination, Nat. Biotechnol., 1998, 16, 49-58.

6 D. J. Maxwell, J. R. Taylor and S. Nie, Self-assembled nanoparticle probes for recognition and detection of biomolecules, J. Am. Chem. Soc., 2002, 124, 9606-9612.

7 A. Tsourkas and G. Bao, Shedding light on health and disease using molecular beacons, Brief Funct. Genomic Proteomic, 2003, 1, 372384.

8 L. G. Kostrikis, S. Tyagi, M. M. Mhlanga, D. D. Ho and F. R. Kramer, Molecular beacons: Spectral genotyping of human alleles, Science, 1998, 279, 1228-1229.

9 S. A. E. Marras, F. R. Kramer and S. Tyagi, Genotyping single nucleotide polymorphisms with molecular beacons, The Humana Press Inc., Towada, NJ, 2003, vol. 212, pp. 111-128.

10 S. S.-M. Sum, D. K.-H. Wong, M.-F. Yuen, H.-J. Yuan, J. Yu, C.-L. Lai, D. Ho and L. Zhang, Real-time PCR assay using molecular beacon for quantitation of hepatitis B virus DNA, J. Clin. Microbiol., 2004, 42, 3438-3440.

11 S. R. Lewin, M. Vesanen, L. Kostrikis, A. Hurley, M. Duran, L. Zhang, D. D. Ho and M. Markowitz, Use of real-time PCR and molecular beacons to detect virus replication in Human Immunodeficiency Virus type 1-infected individuals on prolonged effective antiretroviral therapy, J. Virol., 1999, 73, 6099-6103.

12 J. A. M. Vet, A. R. Majithia, S. A. E. Marras, S. Tyagi, S. Dube, B. J. Poiesz and F. R. Kramer, Multiplex detection of four pathogenic retroviruses using molecular beacons, Proc. Natl. Acad. Sci. U. S. A., 1999, 96, 6394-6399.

13 M. Varma-Basil, H. El-Hajj, S. A. E. Marras, M. H. Hazbon, J. M. Mann, N. D. Connell, F. R. Kramer and D. Alland, Molecular beacons for multiplex detection of four bacterial bioterrorism agents, Clinical Chem., 2004, 50, 1060-1063. 
14 D. P. Bratu, B.-J. Cha, M. M. Mhlanga, F. R. Kramer and S. Tyagi, Visualizing the distribution and transport of mRNAs in living cells, Proc. Natl. Acad. Sci. U. S. A., 2003, 100, 13308-13313.

15 P. J. Santangelo, B. Nix, A. Tsourkas and G. Bao, Dual FRET molecular beacons for mRNA detection in living cells, Nucleic Acids Res., 2004, 32, e57.

16 W. Tan, K. Wang and T. J. Drake, Molecular beacons, Curr. Opin. Chem. Biol., 2004, 8, 547-553.

17 J. B. Biggins, J. R. Prudent, D. J. Marshall, M. Ruppen and J. S. Thorson, A continuous assay for DNA cleavage: The application of "break lights" to enediynes, iron-dependent agents, and nucleases, Proc. Natl. Acad. Sci. U. S. A., 2000, 97, 13537-13542.

18 S. Jockusch, A. A. Martí, N. J. Turro, Z. Li, X. Li, J. Ju, N. Stevens and D. L. Akins, Spectroscopic investigation of a FRET molecular beacon containing two fluorophores for probing DNA/RNA sequences, Photochem. Photobiol. Sci., 2006, 5, 493-498.

19 P. Zhang, T. Beck and W. Tan, Design of a Molecular Beacon DNA Probe with Two Fluorophores, Angew. Chem., Int. Ed., 2001, 40, 402405 .
20 A. A. Martí, S. Jockusch, Z. Li, J. Ju and N. J. Turro, Molecular Beacons with intrinsically fluorescent nucleotides, Nucleic Acids Res., 2006, 34, e50.

21 A. K. Tong, S. Jockusch, Z. Li, H.-R. Zhu, D. L. Akins, N. J. Turro and J. Ju, Triple Fluorescence Energy Transfer in Covalently TriChromophore-Labeled DNA, J. Am. Chem. Soc., 2001, 123, $12923-$ 12924.

22 A. K. Tong, Z. Li, G. S. Jones, J. J. Russo and J. Ju, Combinatorial fluorescence energy transfer tags for multiplex biological assays, Nat. Biotechnol., 2001, 19, 756-759.

23 A. K. Tong and J. Ju, Single nucleotide polymorphism detection by combinatorial fluorescence energy transfer tags and biotinylated dideoxynucleotides, Nucleic Acids Res., 2002, 30, e19.

24 J.-F. Brunet, E. Shapiro, S. A. Foster, E. R. Kandel and Y. Iino, Identification of a peptide specific for Aplysia sensory neurons by PCRbased differential screening, Science, 1991, 252, 856-859.

25 J. R. Lakowicz, Topics in Fluorescence Spectroscopy, Volume 5: Nonlinear and Two-Photon-Induced Fluorescence, Plenum Press, New York, 1997, vol. 5, 544 pp. 